\title{
The Effects of Whole Body Vibration on the Limits of Stability in Adults With Subacute Ankle Injury
}

\author{
Sonia Young, PT, DPT, EdD, NCS ${ }^{1}$, , Harvey W. Wallmann, PT, DSc, ATC, CSCS, SCS Emeritus², Kailey L. Quiambao, PT, \\ $\mathrm{DPT}^{3}$, Brooke M. Grimes, PT, DPT ${ }^{4}$ \\ ${ }^{1}$ Western Kentucky University, Bowling Green, KY, USA, ${ }^{2}$ University of Findlay, Findlay, OH, USA, ${ }^{3}$ Drayer Physical Therapy, Hilliard, OH, USA, ${ }^{4}$ KORT \\ Physical Therapy, Glasgow, KY, USA \\ Keywords: movement system, whole body vibration, subacute, limits of stability, balance, ankle sprain \\ https://doi.org/10.26603/001c.24250
}

\section{International Journal of Sports Physical Therapy}

Vol. 16, Issue 3, 2021

\section{Background}

Limited research exists on the effects of both high and low frequency whole body vibration (WBV) on individuals with subacute lateral ankle sprains.

\section{Hypothesis/Purpose}

To examine the difference in the effects of high and low frequency WBV on limits of stability (LOS) in adults with a subacute ankle sprain. It was hypothesized that WBV would improve effects on outcome variables for LOS as a component of dynamic balance.

\section{Study Design}

Quasi-experimental, pretest-posttest design.

\section{Methods}

Fifteen participants ages 19-27 years (Mean age 22ะ2.36) with either a Grade I or Grade II lateral ankle sprain received WBV in bilateral stance under three randomized conditions (high frequency-25 Hz, low frequency-6 Hz, and control, which consisted of bilateral stance with machine off) for six minutes over three sessions (one time per week). The LOS test, consisting of 5 variables, were assessed using the NeuroCom ${ }^{\circledR}$ Balance Manager-SMART EquiTest ${ }^{\circledR}$ (Natus Medical Incorporated, Pleasanton, CA) at baseline and after the intervention period. The participants completed a practice LOS test and then had a six-minute standing rest break. After the rest break, they completed the pre-LOS (baseline) test. Intervention was administered using the Galileo ${ }^{\circledR}$ Med L Chip Research (Novotec Medical $\mathrm{GmbH}$, Pforzheim, Germany) for six minutes for the appropriate condition of either high or low frequency WBV or control. Data analysis was performed using 2-Way (2x3) Repeated Measures ANOVAs with additional post hoc testing as needed.

\section{Results}

Significant interactions were found for reaction time (RT), movement velocity (MVL), and maximal excursion (MXE) composite scores with a decrease in RT of 0.117 seconds $(p=0.022)$ between control and high frequency conditions during the post LOS. For composite MVL, an increase of 0.547 degrees/second $(\mathrm{p}=0.002)$ between pre- and

Corresponding author:

Sonia N. Young, PT, DPT, EdD, NCS

Associate Professor

Doctor of Physical Therapy Program

College of Health and Human Services, Western Kentucky University

1906 College Heights Blvd. \#21031

Bowling Green, KY 42101-1031

Phone: (270) 745-3233

Fax: (270) 745-3497

E-mail: sonia.young@wku.edu 
post-high frequency WBV occurred. For composite MXE, an increase of $2.13 \% \mathrm{p}=0.031$ (when comparing pre- and post-high frequency WBV.

\section{Conclusion}

Findings suggest that a single session of high frequency WBV in individuals with a subacute lateral ankle sprain may result in improvement in several components of postural stability. WBV is a quick intervention that could be implemented in physical therapy clinics, athletic training rooms, and workout facilities to improve an individual's LOS as a component of dynamic balance one to eight weeks post lateral ankle sprain.

\section{Level of Evidence}

$2 b$

\section{INTRODUCTION}

Ankle sprains are a common sports related injury with about 23,000 occurring daily in the United States. ${ }^{1,2} \mathrm{Al}-$ though ankle sprains occur in many types of sports, the most common sports are basketball, football, and soccer. There are three types of ankle sprains: medial (involves the deltoid ligament), lateral (involves the anterior talofibular ligament, calcaneofibular, and posterior talofibular), and syndesmotic, also called a high ankle sprain (involves the interosseous ligament between the distal tibia and fibula). Lateral ankle sprains, being the most common type of sprain, occur from either an uncontrolled force or from excessive supination and adduction when the ankle is plantarflexed. ${ }^{1}$ Ankle injuries are also defined along a continuum of acute (immediately post injury to 4 days post), subacute ( 1 to 8 weeks post injury), and chronic (greater than 8 weeks post). ${ }^{3}$ The severity of ankle sprains are defined by Grades I through III which note the percentage of tissue that is torn or disrupted. Grade I is defined by less than $25 \%$ of ligament fibers torn, Grade II is defined as $25 \%$ to $75 \%$ of ligament fibers torn, and Grade III is defined as greater than $75 \%$ of the ligament being non-contiguous. ${ }^{4,5}$

This study focused on the subacute phase of an ankle sprain which is defined as one to eight weeks after the initial injury. ${ }^{3}$ Certain special tests can be performed clinically to determine the grade of ankle sprain. The most common special tests used are the anterior drawer test and the talar tilt test. ${ }^{4-6}$ A Grade I sprain is classified by a positive scoring on the anterior drawer test and a negative scoring on the talar tilt test, which means there is damage to the anterior talofibular ligament. A Grade II is classified by a positive scoring on both the anterior drawer test and the talar tilt test, which means there is damage to both the anterior talofibular ligament and the calcaneofibular ligament. ${ }^{4,6-8}$ Ankle sprains may impact various components of the movement system, namely the musculoskeletal and nervous systems. Not only do ankle sprains damage ligaments, but they also damage mechanoreceptors and the joint capsule, which can then potentially adversely affect joint proprioception and balance. Most common treatments for ankle sprains initially involve the following: taping, bracing, protection, rest, ice, compression, and elevation. General rehabilitation typically involves the use of range of motion (ROM), strength training, and balance/proprioception training in order to progress the individual. Another rehabilitation technique includes the use of whole body vibration (WBV). Previous research has been conducted on the effects of WBV as a rehabilitation intervention on chronic ankle instability (CAI); however, there is limited research on the effects of WBV as a rehabilitation intervention on subacute ankle sprains. ${ }^{1}$

WBV is a modality that is recognized for improving neurovascular and neuromuscular performance and balance through vertical sinusoidal oscillations. ${ }^{9}$ The vibration moves from the foot throughout the rest of the body. ${ }^{9}$ There are several different vibrations that can be utilized, such as an alternating, simultaneous, and multidirectional. ${ }^{9}$ The frequencies range from low to high depending on the type of vibration administered. For alternating side-to-side vertical WBV, low frequency is between 5-12 hertz $(\mathrm{Hz})$ and high frequency is between $20-30 \mathrm{~Hz} .{ }^{10}$ For simultaneous vertical WBV, low frequency is at $20-30 \mathrm{~Hz}$ and high frequency is $35-50 \mathrm{~Hz} .{ }^{9}$ Frequencies below $12 \mathrm{~Hz}$ cause muscle contraction without triggering the stretch reflex resulting in a conscious compensation of movement, whereas high frequencies focus on training muscle power and force through the rapid contraction and partial relaxation of the muscle. ${ }^{10} \mathrm{WBV}$ has been shown to improve performance and balance in sub-populations such as the elderly and individuals with motor impairments like Parkinson Disease, stroke, and multiple sclerosis. ${ }^{11}$ However, Ritzmann et al. have recently suggested that WBV can enhance balance control in young healthy individuals. ${ }^{11}$ Research has also shown that WBV can lead to physiological changes in muscle spindles, joint mechanoreceptors, strength, and power resulting in enhanced proprioception and balance in individuals with CAI. ${ }^{12}$ Although high frequencies have been shown to increase neurovascular and neuromuscular performance and balance, there has been insufficient research that examines the effects of low frequency WBV on the limits of stability (LOS) as a component of dynamic balance of young, healthy or injured adults, particularly examining the effects of WBV on subjects who have sustained Grade I and II ankle injuries, subacutely.

Postural stability is the ability to maintain one's center of gravity (COG) within the base of support (BOS); the maximum range in which the COG can be displaced without loss of balance or changing the BOS is referred to as the LOS. ${ }^{13,14}$ The LOS test measures a person's reaction time (RT), movement velocity (MVL), endpoint excursion (EPE), maximal excursion (MXE), and directional control (DCL) when shifting their COG in eight different directions. ${ }^{14,15}$ At the two ends of the lifespan, young children and older adults, postural stability is at its lowest. ${ }^{16}$ Young adults, however, have a larger amount of postural stability and are 
able to expand their LOS, which decreases their risk of falls. Young adults that participate in athletics depend on their LOS to maintain their balance while performing dynamic movements such as: throwing, catching, kicking, and blocking. Injury to ankle ligaments will decrease an individual's LOS due to swelling, pain, and instability. Previous research has used the LOS test to determine its effects on balance in individuals who have sustained ankle sprains. ${ }^{17}$

The purpose of this study was to examine the difference in the effects of high and low frequency WBV on LOS in adults with a subacute ankle sprain. It was hypothesized that WBV would improve effects on outcome variables for LOS as a component of dynamic balance.

\section{METHODS}

\section{RESEARCH DESIGN}

Quasi-experimental pretest-posttest design.

\section{PARTICIPANTS}

Fifteen participants, aged 19-27 years (Mean age 22 \pm 2.36 ) years were recruited through posted flyers on a University campus and through school-wide emails. All 15 participants had sustained a lateral ankle sprain (Grade I Sprain - $\mathrm{n}=12$; Grade II Sprain $-n=3$ ). Those that participated in the study were rewarded a $\$ 25$ gift card after completion of all three sessions through an internal university grant. The inclusion criteria included the following: between the ages of 18 and 29 , having sustained a Grade I or II ankle sprain in the prior one to five weeks, and able to speak and read English. While the subacute phase was recognized as occurring between one to eight weeks post ankle injury, the inclusion criteria was for the prior one to five weeks to ensure data collection could be completed within the subacute timeframe. The exclusion criteria included the following: sustained an ankle injury in the prior six days or longer than eight weeks, had sustained a Grade III ankle sprain, were on medications that affected balance, and/or were currently pregnant. The participants were informed of all the study risks and benefits of receiving WBV prior to filling out a pre-screening questionnaire and then were instructed on how to perform the LOS Test. All participants were required to sign an informed consent prior to participation. The study received university institutional review board approval prior to screening or data collection.

\section{INSTRUMENTATION AND OUTCOME MEASURES}

The Galileo ${ }^{\circledR}$ Med L Chip Research (Galileo) (Novotec Medical GmbH, Pforzheim, Germany), was used to provide the WBV intervention. The Galileo is a guided therapy machine, which provides an alternating side-to-side WBV while stimulating the normal gait pattern. There is a separate control panel that allows the researcher to control the frequency and duration of the intervention and a handrail available for additional participant safety. The vertical lines on the base of the machine allow for consistency of foot placement. High and low frequency WBV were delivered at $25 \mathrm{~Hz}$ and $6 \mathrm{~Hz}$, respectively.

The NeuroCom ${ }^{\circledR}$ Balance Manager system (NeuroCom)

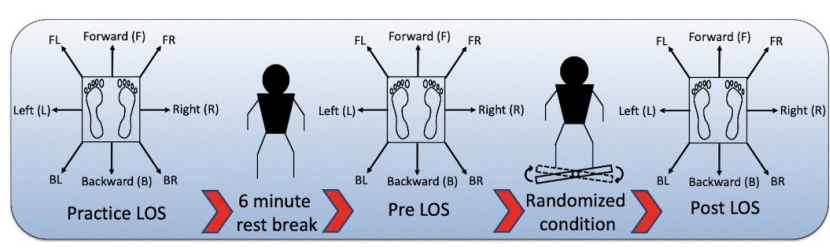

Figure 1: Study Protocol

LOS = Limits of Stability

(SMART EquiTest, Natus Medical Incorporated, Pleasanton, CA) was used to assess balance by the administration of the LOS test to assess the participants' RT, MVL, EPE, MXE, and DCL when shifting their COG in eight different directions. This device also is accompanied with a harness for participant safety. The participants have eight seconds to shift their COG in an effort to move the cursor to its appropriate destination. The eight directions include the following: forward, forward right, right, backward right, backward, backward left, left, and forward left. A composite score was then generated as a compilation of the average of each direction for each variable.

\section{DATA COLLECTION}

Fifteen participants were identified, completed the informed consent and the Pre-Screening Questionnaire, and received either a Grade I or Grade II lateral ankle sprain classification after assessment by a Board-Certified Clinical Specialist in Sports Physical Therapy using the anterior drawer test and the talar tilt test. ${ }^{5}$ This clinician, along with a Board-Certified Clinical Specialist in Neurologic Physical Therapy, provided the supervision and training to two second year physical therapy student examiners who performed data collection. The student examiners administered the LOS tests as well as the WBV intervention. Figure 1 displays the protocol that was followed in the study. Participants attended a single session one time per week for three consecutive weeks. In each session, the participants completed a practice LOS test on the NeuroCom and then had a six-minute standing rest break followed by a pre-LOS (baseline) test and then an intervention followed by a postLOS test. The intervention consisted of receiving WBV utilizing the Galileo in bilateral stance under one of three randomized conditions (high frequency-25 Hz, low frequency- 6 $\mathrm{Hz}$, and control, which consisted of bilateral stance on the Galileo with machine off) for six minutes in a university research lab setting.

Floor markings and scripts were made for every session to allow for standardization. All participants were secured in a harness during the LOS test and the harness remained on for the entirety of each session.

\section{DATA ANALYSIS}

A 2 (time: pretest, posttest) x 3 (condition: low frequency WBV, high frequency WBV, control) ANOVA with repeated measures on both factors was used to determine changes for each of the dependent variables. An alpha level was set at $p<0.05$ and power was set at 0.8 for each variable. Post 
hoc analysis was performed with pairwise comparisons involving repeated measures ANOVAs if significant interactions were found. Statistical analysis was performed using the SPSS, 25.0 statistical software package (IBM Corp. Released 2018. IBM SPSS Statistics for Windows, Version 25.0. Armonk, NY: IBM Corp.).

\section{RESULTS}

Significant interactions for composite scores for the LOS variables of RT, MVL, and MXE were found (Table 1). For composite RT, a significant two-way interaction was noted between condition and time $\mathrm{F}(2,28)=3.505$, $\mathrm{p}=0.044$ (Table 1).

Post hoc analysis for simple main effects for condition revealed significant differences among the three post conditions of LOS (high frequency, low frequency, control) $\mathrm{F}(2,28)=3.754, \mathrm{p}=0.036$ (Figure 2).

Pairwise comparisons revealed a significant decrease in composite RT of 0.117 seconds (95\% CI, .019 to .214, $\mathrm{p}=0.022$ ) (Figure 3 ) between control and high frequency conditions during the post LOS. For composite MVL, a significant two-way interaction was noted between condition and time $\mathrm{F}(2,28)=3.394, \mathrm{p}=0.048$ (Table 1).

Post hoc investigation for simple main effects for time revealed a significant increase in composite MVL of 0.547 degrees/second $\mathrm{F}(1,14)=13.771, \mathrm{p}=0.002$ (95\% CI, .231 to .863) (Figure 4) between pre- and post-high frequency WBV. For composite MXE, a significant two-way interaction was noted between condition and time $\mathrm{F}(2,28)=4.271$, $\mathrm{p}=0.024$ (Table 1). Post hoc investigation for simple main effects for time revealed a significant increase in composite MXE of $2.13 \% \mathrm{~F}(1,14)=5.767, \mathrm{p}=0.031$ (95\% CI, .228 to 4.039) (Figure 5) when comparing pre- and post-high frequency WBV.

\section{DISCUSSION}

The purpose of this study was to examine the difference in the effects of high and low frequency WBV on LOS in adults with a subacute ankle sprain. It was hypothesized that WBV would improve effects on outcome variables for LOS as a component of dynamic balance. Out of the five LOS composite scores assessed, only three of them were statistically significant between interventions. Although low frequency WBV was examined in this study, the results indicate that only high frequency WBV may impact the movement system and lead to improvements in some of the fundamental variables required for postural stability. Regarding clinical meaningfulness, while the current study found statistically significant change occurring in RT (decrease of .117 seconds), MVL (increase of .547 deg/sec), and MXE (increase of $2.13 \%$ ), true, clinically meaningful, change appears to be questionable based on previous research. Alsalaheen et al. examined LOS test composite scores as well as the individual LOS components in healthy adolescents and found a $\mathrm{MDC}_{95}$ of 0.13 seconds for RT, $1.55 \mathrm{deg} / \mathrm{second}$ for MVL, and $5.9 \%$ for MXE in order for true change to occur. ${ }^{18}$ Although these results cannot be generalized to the current study population of those with subacute lateral ankle sprains, it may indicate that clinically meaningful changes did not oc-
Table 1: Limits of Stability Composite Results

\begin{tabular}{|c|c|}
\hline Variable & p value \\
\hline RT & $F(2,28)=3.505, p=0.044^{*}$ \\
\hline MVL & $F(2,28)=3.394, p=0.048^{*}$ \\
\hline EPE & $F(2,28)=1.406, p=0.262$ \\
\hline MXE & $F(2,28)=4.271, p=0.024^{*}$ \\
\hline DCL & $F(2,28)=1.835, p=0.178$ \\
\hline
\end{tabular}

$\mathrm{RT}=$ Reaction Time, MVL $=$ Movement Velocity, $\mathrm{EPE}=$ End Point Excursion, $\mathrm{MXE}=\mathrm{Maxi}-$ mum Excursion, DCL $=$ Directional Control, ${ }^{*}=\mathbf{p}<0.05$

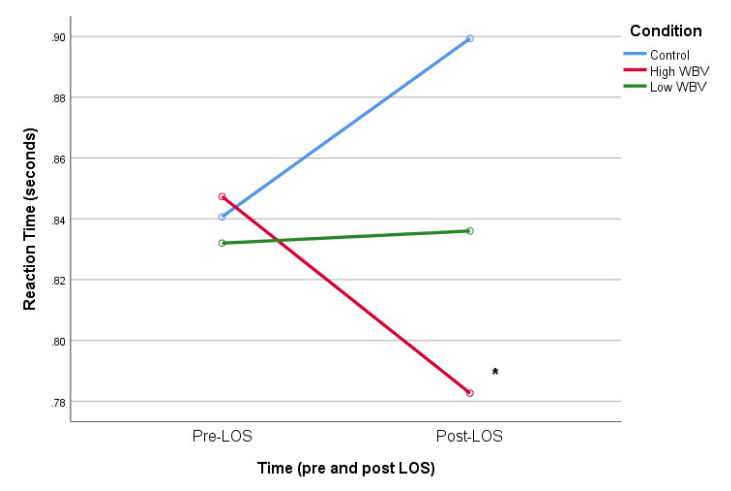

Figure 2: Interaction Effect for Composite Reaction Time

*p=0.036
WBV = Whole Body Vibration

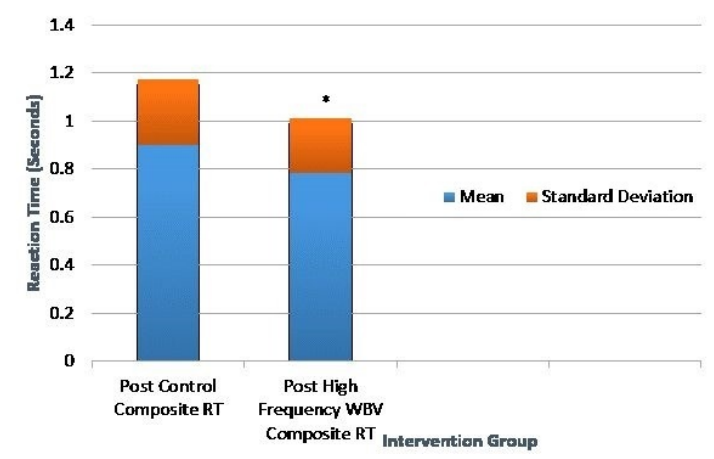

Figure 3: Post Composite Reaction Time

*p=0.022

RT - Reaction Time

WBV - Whole Body Vibration

cur.

\section{HIGH FREQUENCY WBV}

To the authors' knowledge, there are no other current research studies that have examined the effects of WBV on the LOS as a component of dynamic balance in individuals 
with a lateral ankle sprain. However, several authors have examined the effects of WBV on balance in a variety of populations. According to Ritzmann et al., high frequency alternating side-to-side vertical WBV, administered through the Galileo Sport, improved balance control as noted by center of pressure movement during one-leg stance for 38 physically fit adult students who trained for four weeks. ${ }^{11}$ This is consistent with the results of the current study in that high frequency $(25 \mathrm{~Hz})$ WBV delivered in an alternating side-to-side device improved RT, MVL, MXE of the LOS Test that correlate with improved balance in a similar age population. However, the Ritzmann, et al. ${ }^{11}$ study looked at a healthy adult population as compared to the current subacute ankle sprain group. The current study is also in agreement with Sierra-Guzman et al., who found improvements in balance using the Biodex Balance System after high frequency simultaneous vertical WBV $(30-40 \mathrm{~Hz})$ utilizing the Excel Pro vibration platform in 50 recreational athletes with CAI who were randomized to three groups: vibration, nonvibration, or control. ${ }^{19}$ The intervention occurred one time per week for three consecutive weeks. Again, while not addressing subacute ankle injuries and with using simultaneous vertical rather than alternating side-to-side WBV, those authors found that elements of balance also improved in CAI when utilizing high frequency WBV.

Additionally, while not addressing balance specifically, other studies have examined the impact of WBV on individuals with CAI and found high frequency WBV to improve outcomes related to range of motion, strength, and proprioception. One such study had 39 participants with CAI randomized to three groups: normative, static stretch, and static stretch with WBV (simultaneous vertical) at $34 \mathrm{~Hz}^{20}$ The study was conducted four days per week for three weeks. The static stretching with high frequency WBV group saw increases with dorsiflexion ROM that were better than with static stretching alone. ${ }^{20}$ While that study investigated individuals with chronic conditions and not subacute ankle injuries, it is interesting to note that high frequency WBV was beneficial to treatment in a CAI population. Likewise, the present study found that high frequency WBV may be beneficial to the subacute ankle injury population. The reasons for this benefit may be two-fold. As previously mentioned, previous researchers have found that WBV produces physiological changes in muscle spindles, joint mechanoreceptors, strength, and power resulting in enhanced proprioception and balance. ${ }^{12}$ As such, one reason may involve lowering the threshold for activation of these receptors. Additionally, high frequency WBV may produce a summative effect of the stimulus required to produce activation in these structures.

\section{LOW FREQUENCY VIBRATION}

Low frequency $(6 \mathrm{~Hz})$ alternating side-to-side WBV was not found to have a significant impact on balance in an adult population with subacute ankle sprains in the current study. Additionally, there are no studies to date showing that low frequency WBV has a favorable impact on dynamic balance for a population suffering from subacute lateral ankle sprains.

However, the results of the current study are in line with

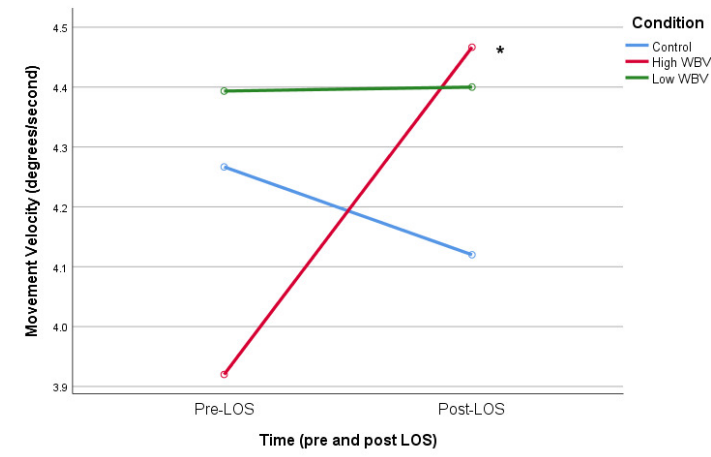

\section{Figure 4: Interaction Effect for Composite Movement Velocity}

* $\mathbf{p}=\mathbf{0 . 0 0 2}$

WBV - Whole Body Vibration

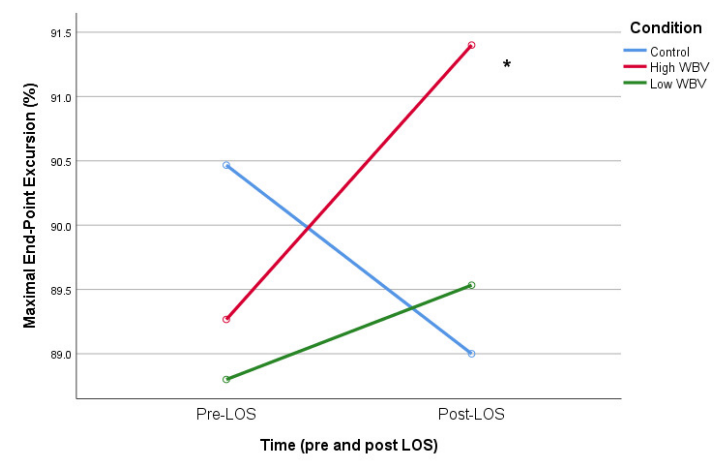

Figure 5: Interaction Effect for Composite Maximal Excursion

*p=0.031

WBV - Whole Body Vibration

the results of a study by Rendos et al. in which 19 active adults were studied in a control group and a group with CAI. ${ }^{12}$ The participants received low frequency $(30 \mathrm{~Hz})$ simultaneous vertical WBV and sham interventions on 3 different testing days in a random order. Results indicated that the low frequency WBV did not improve static or dynamic balance in either group. ${ }^{12}$

\section{LIMITATIONS}

Limitations in the present study were noted. For one, spontaneous healing over time may have been a factor. All participants completed three sessions over the course of three weeks. Although all participants remained in the subacute phase of injury during their participation in this study, the healing process for their ankle injury was continuous, and likely variable among subjects. Thus, it is possible that spontaneous healing over the study period could have enhanced the effects of the intervention, and occurred differently among subjects.

Second, a learning effect could have influenced the results. All participants were required to perform a practice LOS Test to allow each participant to acclimate to the test. 
Therefore, as participants continued through the sessions, they may have demonstrated improvements in their preand post- LOS testing secondary to this effect.

Finally, participants may not have maintained a consistent knee position during the intervention. Knee flexion was measured using a goniometer once every session, as the participant mounted the Galileo, to ensure a standardization of each participant's stance during the randomized condition portion of the session. Every participant was then verbally instructed to maintain this set degree of knee flexion throughout receiving the random condition. However, knee flexion was never re-assessed throughout the session and may have changed, potentially altering the effects of WBV.

\section{CONCLUSIONS}

The results of the current study suggest that a single session of high frequency WBV for individuals with a sub-acute ankle sprain resulted in statistically significantly improved RT, MVL, and MXE during the post-LOS test performance. There were no significant findings associated with either the low frequency WBV condition or the control condition. Further research is required to determine the clinical significance of these results and to investigate the long-term effects of a single session of high frequency WBV, since this study focused mainly on the immediate effects. Additional research should also be performed to determine if multiple sessions of high frequency WBV over the course of several weeks would be more beneficial in improving additional variables of an individual's LOS. Moreover, a comparison between the effects of alternative side-to-side delivery of WBV and simultaneous vertical WBV on individuals with subacute ankle injuries would be beneficial.

\section{CONFLICT OF INTEREST}

The authors report none.

Submitted: July 22, 2020 CDT, Accepted: November 08, 2020

CDT 


\section{REFERENCES}

1. Granado M, Matlick D. Ankle sprain. Richman S, ed. CINAHL Rehabil Guide. January 2018.

2. Kemler E, van de Port I, Backx F, van Dijk CN. A systematic review on the treatment of acute ankle sprain: brace versus other functional treatment types. Sports Med Auckl NZ. 2011;41(3):185-197. doi:10.216 5/11584370-000000000-00000

3. Terada M, Pietrosimone BG, Gribble PA. Therapeutic interventions for increasing ankle dorsiflexion after ankle sprain: a systematic review. $J$ Athl Train. 2013;48(5):696-709. doi:10.4085/1062-605 $\underline{0-48.4 .11}$

4. Birrer RB, Fani-Salek MH, Totten VY, Herman LM, Politi V. Managing ankle injuries in the emergency department. J Emerg Med. 1999;17(4):651-660. doi:1 0.1016/s0736-4679(99)00060-8

5. Martin RL, Davenport TE, Paulseth S, Wukich DK, Godges JJ. Ankle stability and movement coordination impairments: ankle ligament sprains. J Orthop Sports Phys Ther. 2013;43(9):A1-40. doi:10.2519/jospt.201 $\underline{3.0305}$

6. Rubin A, Sallis R. Evaluation and diagnosis of ankle injuries. Am Fam Physician. 1996;54(5):1609-1618.

7. Polzer H, Kanz KG, Prall WC, et al. Diagnosis and treatment of acute ankle injuries: development of an evidence-based algorithm. Orthop Rev. 2012;4(1):e5. doi:10.4081/or.2012.e5

8. Chan KW, Ding BC, Mroczek KJ. Acute and chronic lateral ankle instability in the athlete. Bull NYU Hosp Jt Dis. 2011;69(1):17-26.

9. Chanou K, Gerodimos V, Karatrantou K, Jamurtas A. Whole-body vibration and rehabilitation of chronic diseases: A review of the literature. J Sports Sci Med. 2012;11(2):187-200.

10. Frequency ranges of Galileo training and training objectives - Novotec Medical. https://www.galileo-tra ining.com/us-english/products/galileo-therapy-syste ms/background/frequencies.html. Accessed March 7, 2017.
11. Ritzmann R, Kramer A, Bernhardt S, Gollhofer A. Whole body vibration training - improving balance control and muscle endurance. PLOS ONE. 2014;9(2). doi:10.1371/journal.pone.0089905

12. Rendos NK, Jun H-P, Pickett NM, et al. Acute effects of whole body vibration on balance in persons with and without chronic ankle instability. Res Sports Med Print. 2017;25(4):391-407. doi:10.1080/1543862 7.2017.1365299

13. Alexander NB. Postural Control in Older Adults. J Am Geriatr Soc. 1994;42:93-108.

14. Pickerill ML, Harter RA. Validity and reliability of limits-of-stability testing: a comparison of 2 postural stability evaluation devices. J Athl Train. 2011;46(6):600-606.

15. Wallmann HW, Player K, Bugnet M. Acute effects of static stretching on balance in young versus elderly adults. Phys Occup Ther Geriatr. 2012;30(4):301-315.

16. Schwesig R, Fischer D, Kluttig A. Are there changes in postural regulation across the lifespan? Somatosens Mot Res. 2013;30(4):167-174. doi:10.3109/ 08990220.2013 .779245

17. Dallas G, Dallas K. Effects of ankle joint injuries on balance in male and female gymnasts. Sci Gymnast J. 2016;8(2):149-156.

18. Alsalaheen B, Haines J, Yorke A, Broglio SP. Reliability and construct validity of limits of stability test in adolescents using a portable forceplate system. Arc of Phys Med and Rehab. 2015;96(12):2194-2200. $\underline{\mathrm{d}}$ oi:10.1016/j.apmr.2015.08.418

19. Sierra-Guzmán R, Jiménez-Diaz F, Ramírez C, Esteban P, Abián-Vicén J. Whole-body-vibration training and balance in recreational athletes with chronic ankle instability. J Athl Train. 2018;53(4):355-363. doi:10.4085/1062-6050-547-16

20. Feland JB, Thalman L, Hunter I, Cochrane DJ, Hopkins JT. Whole-body vibration and stretching enhances dorsiflexion range of motion in individuals with chronic ankle instability. Phys Ther Sport. 2020;44:1-7. doi:10.1016/i.ptsp.2020.04.001 